\title{
Explore the Harmony in Chinese Contemporary Choral Art—"CCTV National Young Singer Grand Prix" as an Example
}

\author{
Lu Jingyuan, Lee Chie Tsang Isaiah
}

\begin{abstract}
The history of the chorus in China can be traced back to the late 20th century and has been developing rapidly. The "CCTV National Young Singer Grand Prix," as one of the top competitions of Chinese singing art since 1984, has actively promoted chorus culture, providing an important platform where chorus groups can appreciate and learn. This paper explores the process of its development and the way of harmony of the chorus in China. The paper is based on synergism theory and takes the "CCTV National Young Singer Grand Prix" as the research object. Through personal observation, more than ten works including "Chun Xiao" "Dong Zu Da Ge", "Meng Gu Xue", etc. will be analyzed through the singing technique, singing method, singing synergism. The possibility of harmony and collision of different natures of chorus art is obtained, expecting to improve the spirituality of chorus and provide a theoretical and practical reference for the majority of chorus art workers.
\end{abstract}

Index Terms: Chorus; Singing method; Synergism

\section{INTRODUCTION}

The Chinese choral art entered the stage of rapid development and prosperity from the 1980s(Yang, F. 2012). Since its development, the chorus groups of various forms and scales ${ }^{1}$ have gradually developed from a simple chorus form to a certain level of professional, artistic and appreciative choral art form today. The "CCTV National Youth Singer Grand Prix" (Qingge Contest) was founded in 1984. As one of the top events in Chinese singing art, in 2008 it officially launched a chorus event, which caught the attention of hundreds of millions of viewers, and is a feast of Chinese choral art collision and display. It is of great significance to improve the performance level of Chinese chorus and the appreciation level of the chorus. The grand contest attracts local professional and non-professional chorus groups from different regions. Professional chorus groups include the General Political Choir of the People's Liberation Army, the Chorus of the Art Academy of the People's Liberation Army, the Choir of the Tianjin Conservatory of Music, the Choir of the Xi'an Conservatory of Music, the Men's Choir of the Chinese Armed Police Corps, the Inner Mongolia Youth Choir, the Dai Song

Revised Manuscript Received on September 22, 2019.

Lu Jingyuan, Faculty of Humanities, Arts and Heritage Universiti Malaysia Sabah, 88400kota Kinabalu, Sabah, Malaysia, lujingyuan79@foxmail.com

Lee Chie Tsang Isaiah, Faculty of Humanities, Arts and Heritage Universiti Malaysia Sabah, 88400kota Kinabalu, Sabah, Malaysia.
Choir, the Second Artillery Tianda Choir and over thirty provincial and central representative teams. The grand contest also invited many famous conductors, composers, and writers as CCTV live judges for two months. These prominent figures include Cao Ding, Li Xincao, Yang Hongnian, Zhao Jiping, Gu Jianfen, Yu Qiuyu, and Yan Su.

This article explores the development of Chinese modern choral art by analyzing its development in the excellent choral works of Qingge Contest. The author's research focuses on the synergy of the chorus and chorus singing technique. The chorus singing method, through the different nature of the choral art harmony and the possibility of collision, enhances the rich spirituality of the chorus.

\section{CHORUS SYNERGY}

The theory of synergy is a systematic science theory founded by the famous German physicist Professor Hermann Haken. This theory holds that the system is omnipresent and all-encompassing. Any single thing is not a system, but a sub-system, and there is nothing that can escape from the system or exist independently (Haken, H. 1997). Anything that leaves one system will fall into another system. The synergistic theory of chorus refers to the full play of the aesthetic function of choral art based on the theory of synergy. This brings about the synchronous and complementary elements between the chorus and the thought, culture, and psychology. These synchronous and complementary elements will optimize the overall function of the chorus, which surpasses the sum of the parts. We will give full play to people's imagination and creativity. Chorus synergy means that each subsystem can cooperate, infiltrate each other, complement each other, and promote the interactive movement between the various subsystems. The orderly result of synergy is a high-level summary of this principle.

The principle of synergy tells us that three basic conditions are needed to generate synergy within the system: first, the system is open and free; second, each subsystem within the system must have a strong unpredictability; third, there are widespread rises and falls in the system ( Haken, $\mathrm{H}$. $\&$ Guo, Z. 1983). If we use these three conditions to analyze the chorus of the People's Liberation Army Art Academy, Chun Xiao, we will find the following: 1 . The chorus work is unaccompanied and has a

${ }^{1}$ Early Chinese choral works: "School 
completely traditional style in its structure. 2. Within the whole work, the chorus members, the vocal accompaniment, the conducting gestures and the body language interpretation of the works are impromptu. 3. This work exhibits certain modern popular creative thinking. The tonal aspect reflects the combination of tonality or atonality and the use of improvisational elements to portray the background of the music. This includes the use of vocals to create natural sound effects such as imitating bird sounds, the sounds of Beijing Opera singers and the noises of crowded environments.

\section{CHORUS TECHNIQUE}

The use of the concept of synergy between chorus subsystems is an important way to pursue harmony in the chorus. The beauty of harmony between man and nature, the pursuit of man to find the "similarity or dissimilarity" is the aesthetic feature of oriental art. It is the aesthetic characteristics pursued by Chinese contemporary choral art (Xie, L. 2005). In a chorus competition, we can do a good job of incorporating various systems within the chorus, which shows a good combination of the various environments around the competition. However, there is a high level of difficulty, especially when some works require singing technique, language, and artistic performance skills. The synergy of sound, language, pitch and vocal method becomes more and more important.

\section{A. Voice}

The development of chorus voice training and singing methods in countries around the world is based on the Italian vocal method (Ma, G. 2002). From the perspective of legacy and development, China is no exception. Almost all of the voices of the national young singer TV contest chorus are based on the Italian vocal method, which is based on mixed sound and half-sound. For example, General Political Choir, Xi'an Conservatory of Music Choir, Tianjin University Choir, Northwest University for Nationalities, Harbin Teacher's University Music School Boys Choir, Nanjing School of Art "Meng" Girls Choir, Shenyang Conservatory of Music Northern Women's Folk Song Choir, China Armed Police Boys Choir, Yunnan "Small Wells" Miao Peasant Choir, Sichuan Conservatory of Music Choir, and also, the Beijing Language University Chinese and foreign students choir.

According to our personal observation, the singing voice of the chorus group in a grand contest can be summarized into three characteristics: the performance of the first voice is to adopt the original ethnic singing method, such as the chorus of Inner Mongolia, and the "Dong $\mathrm{Zu} \mathrm{Da} \mathrm{Ge".} \mathrm{The}$ singers are all in national choirs, and the voices are unadulterated, simple and pure. The second voice is close to modern pop music such as jazz. The third is the voice of foreign classical singing and Chinese traditional singing. Most of the singers are professional groups, music academies, and college choirs. They all express the characteristics of the classical chorus in the East and West: they are soft, lyrical, beautiful, harmonious, mostly vocal and semi-acoustic.

\section{B. Vocal Method}

The vocal method of the choruses in the grand contest is diverse. Due to its multi-voice characteristic, the vocal method is based on the chorus works themselves and the singing ability of their singers. However, the development of vocalization method is according to guidance or conduct, and the performance ability, as well as the overall, working together of the chorus (Yang, H. 2002). In order to achieve the unity, harmony, and overall balance and special effects of the voice, the choirs who were selected to participate in the National Youth Song Competition invited famous national conductors and vocal guides. For example, the artistic director of the Inner Mongolia Youth Choir is the conductor Aaron Göhler. The Haizheng Song and Dance Troupe invited art director Meng Dapeng, while the People's Liberation Army Art Academy invited Sang Yesong, the famous conductor. Peiyang Chorus of Tianjin University invited Ren Baoping, Wu Lingfen, etc. To train, such as in circulatory breathing, and the use of the overall chest and abdomen breathing method. In the rehearsal, they had strict requirements of a vocal method for each chorus member. We have seen multiple voices in the rehearsals, such as the "Cannon" and "polyphonic" vocalization method, "soft and harmony" vocalization method, and vocal practice method specially formulated for different singing methods. For example, the Inner Mongolia vocal method uses the long tone of primitive freedom as the basis of the chorus. "Dong $\mathrm{Zu} \mathrm{DaGe^{ \prime 2 }}$ is a "second-level vocalization". This chorus inherits the traditional culture of the Dong ethnic group. Using various vocal methods can lay a foundation of vocal range and shades of sound for many chorus groups.

\section{Pitch}

A chorus depends on the ideal tone, good pitch, and accurate rhythm (Yang Hongnian, 1982). The most basic of these is the requirement for pitch. Since the chorus works more often with multi-voice elements, the pitch is often subject to a variety of factors such as speed, rhythm, harmony and melody constraints.

\section{1) Speed}

Under normal circumstances, the singing speed can be too fast, and the emotions are easily excited, causing emotional and intellectual imbalance. If not controlled, it will cause the vocal organs to be nervous, the breath to be too shallow, and the pitch too high. On the contrary, if the speed is very slow and becomes weak, the breath lacks support, the vocal organs are too loose, and the pitch can easily below. (Shi, Q. 2000) For example, the Choir of Industrial and Commercial College in Hebei University sang "Hebei Laoxiang", which is the adaptation of "Hebei Bangzi". Its melody span is very large ( 5 degrees or more). The speed and melody span of the

In China, most of the multi-voice choruses have been passed down orally from generation to generation. They imitate the sound of birds, insects, mountains and waters, and nature. The number of singers can bos âzens, Erondreds or even thousands, and there is great momentum in thie singing. Led by mezzo soprano, everyone sings. Thanks to the \& rigorous training and tacit understanding of the singers during the perfortance, they flow as one. 
chorus inevitably affected the pitch.

\section{2) Rhythm}

The rhythm plays an important role in the pitch. In the case where the voice is constant, the pitch is generally not easy to stabilize. For example, When the Blue Power Chorus sang "As Long as I Have Music"3, the rhythm of the whole chorus affected the singing of the whole group. The chorus consists of a rhythm which is alternating in length and is a combination of tension and relaxation. During the performance, the members of the team are not aware of the dynamic factors that affected the rhythm, so it was easy to reach an unstable pitch. Therefore, a good pitch is associated with a good sense of rhythm. When we grasp the core of the rhythm, we will grasp the accurate pitch.

\section{3) Harmony}

Chorus is composed of multi-voice elements. When there is a frequent transition of voice, the pitch is most likely to be unstable at this time. The premise upon which a singer sings well is by mastering the harmony of the pitch, as this helps the singer to achieve a stabilized pitch (Yang, H. 1982). When the harmony intensity changes, it is often necessary to make adjustments in the pitch so that the chorus members experience a sense of harmony. The feelings of harmony have a direct impact on the chorus pitch, especially in the process of singing when the harmony changes, the singer needs to pay great attention to the pitch. The following two are the most common cases. The non-acoustic part does not match the acoustic part so that the overall harmony lacks the artistic appeal of the sound. The non-acoustic part is affected by the change of the acoustic part, which has a negative effect on the overall harmony. However, in the chorus contest, the harmony effect produced by the acoustic and non-acoustic part made us marvel at the whole chorus group.

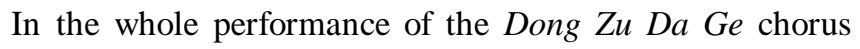
group. the harmony was very obvious. We could hear the effect of the two harmonious sounds, which were very pure. In the case where one of the voices did not change its pitch, the other voice was alternately converted into a first degree and a second-degree voice. This produced the effects of a collision between harmony and disharmony. The fierce collision between the sound effects could be heard up to a few minutes. In the choral art at that time, such a subtle design of the harmony effect was rarely heard. It is really beautiful. The changes that happen for a long period in the second level of voice do not interfere with each other, thus the difficulty level is quite high. Generally speaking, when the differences in the second voice are sung, the non-acoustic part will be affected by the change of the acoustic part. These few minutes of effects which brings about a stable harmony can only be honed in a few years. The song "The song of cicada in May" 4of the Dong Zu Da Ge maintained an amazing harmony effect in the singing, and the collision between harmony and disharmony added a greater artistic appeal to the song.

\footnotetext{
${ }^{3}$ In order to improve the difficulty of the work, a composer adopts a very complicated type of modern pop rhythm.

4"The song of cicada in May", Guizhou TV station selected works, bronze medal of the Qingge Choral Competition.
}

\section{Relativeness Of Pitch}

In chorus singing, the pitch problem can be listed as one of the primary factors to ensure the quality of the chorus. The relative pitch concept generally plays a stable role in the pitch of the acappella. We cannot always talk about physics when explaining the pitch of the chorus. We cannot talk about the "absolutely unchanged" pitch concept. That is to say; the so-called "absolute accuracy" without harmony is meaningless in practice. For example, there are many acappella choruses in the contest. We all know that an acappella chorus mainly relies on vocals or some instruments to perform. The relative stability of the pitch in the performance is one of the most important parts of assessing the acappella. For example, Inner Mongolia Youth Choir's "Meng Gu Xue"5 is a very difficult chorus work. The beginning part is entered with unaccompanied vocals. During the singing, the voices are alternately changed. The pitch between the parts is slightly heard. There was deviation but was soon adjusted to the normal state by the relative polarity of the pitch. This is the characteristic of the prominent chorus group. The chorus part of the song showed the working together of the matouqin and the vocal part. This collision of the characteristic musical instrument and the vocal depicted the innate fusion of the Mongolian people's natural sounds and the vast and beautiful grassland. Finally, returning to the unaccompanied vocals, the vocal singing is more difficult at this time.

This is because the whole chorus is based on the voice part, imitating the sound of the horse galloping on the grassland, the sound from far and near, and from high to low. It is a good use of the relative characteristics of the pitch. We can hear the human voice depicting the picture of nature.

\section{E. The Language Of Singing}

The language of singing is the product of the combination of literary language and musical language. Language is the basis of singing. (Wang, S. 2006) There are many Chinese chorus languages. Language understanding and accurate pronunciation is an important part of chorus. The characteristics of multi-ethnic language make Chinese chorus works have a very rich and diverse national style, which plays a very important role in the formation and development of Chinese chorus style. It has become a chorus of multi-language style and singing techniques. Therefore, we must master the chorus language. For example, the Nankai University Student Choir sang "Hunting the Spirit" and used a large number of national vocals, combined with a beautiful melody to express the unique cultural characteristics in northern Shanxi accurately.

The beauty of language is often used in Chinese poetry. Listening to the language of singing will have a beautiful and fascinating feeling. This is Chinese music creators have incorporated poetry into the creation of chorus. For example, Hebei Communication University Choir's "Yi Qin and Lushan Pass" portrayed the characteristics of Chinese poetry language

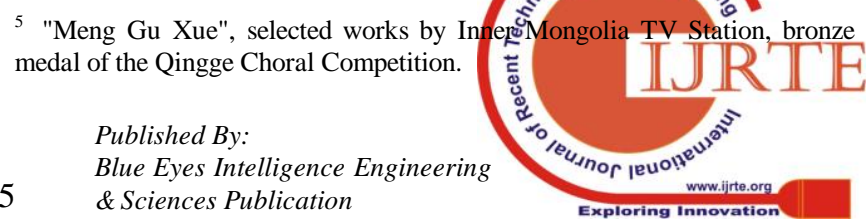


which are the "collecting", "releasing", "urgent" and "slow" of the rhythm and harmony. It is often said that the rounded and harmonious chorus sound, accurate pronunciation combined with training method will make the singing language perfect. Some even adapted folk songs, utilizing its lyrics and words in the chorus, thus highlighting the unique Chinese chorus. Some of these words generally have no practical meaning in the language of singing, but plays a role in depicting language and enriching the content of the works. For example, the "Bao Shu Tune" of the Shandong University Yangge Choir. This is a chorus with lines and words that are based on Shandong folk songs. There are many words in the lyrics; for example, "land", "that", "lengleng", etc. which sound natural and fun. However, some chorus works are very difficult, and the language is quite difficult to grasp. For example, the Balaiji sung by the Peiyang Chorus of Tianjin University. This unaccompanied work is very difficult from the aspects of music, singing, sound techniques, etc. However, the artistic level of the performance of the Peiyang Chorus of Tianjin University is quite prominent. The difficulty level is increased by the difficulty in the change of tone and sound technic. The lead vocal part should have been influenced in terms of singing language and impromptu emotional expression. However, this work has achieved great success in concerts performed abroad. This shows the singing language, singing skills and emotional expression are mutually interactive.

\section{CHORUS SINGING STYLES}

It is well known that there are many styles of chorus singing. Chorus singing depends on the diversity of styles. Chorus singing is mostly based on the singing method. For example, Bel Canto, which has been recognized by the world as a scientific method of vocalization, not only for solo but also for the chorus. There are many singing styles in Bel Canto chorus singing, such as: "musical chorus", "half-voice singing", and "pop chorus". In the "half-voice" method, the singer needs to control the volume of the vocals by singing with a softer voice. This is one of the more popular singing methods in Europe at this stage(Yang, H. 2008). We saw in the contest the "Cultural Song" sung by the Shanxi University Chorus, and "My Sun" sung by the representative team of Zongzheng Zhishu. The Red Star Chorus of the People's Liberation Army Art Academy also sang "He is standing there". All these used the Bel Canto chorus singing style. The overall voice of the chorus is soft, relaxed, and brilliant. Other chorus groups which use various styles of Bel Canto are the Chinese classical chorus "Da Jiangdongqu" by Fujian Normal University Chorus, a Jazz chorus "As Long As I Have Music" by the Blue Power Choir, a religious chorus "Nue Zhenshenqu" by Northeast Normal School Choir and "The Pleasant Poet" by the Chinese University of Mining and Technology Chorus Group.

Contemporary Chinese chorus contains not only profound Western classical chorus singing works but also the "original" chorus singing works of China's natives. The original chorus, through the influence of Western chorus, produced various kinds of styles. The original native chorus

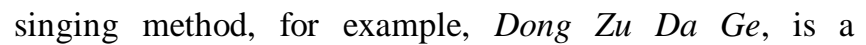

multi-voice performance with no conductor, and no accompaniment and the rhythm is not fixed. This song has a long history in China, and it has been passed down orally, based on the people's memory. The villagers in the whole village formed their singing groups, not only according to the sexes, male and female but also according to different ages. People learned to sing songs. Even from childhood, males and females started to form their singing groups. Upon entering adolescence, the vocal songs between the male and female singers increased, and the difficulty level also gradually increased to the level that we see today. Their voices are like the beauty and splendor of the sky.

There is also a group of ethnic chorus singing. It is the Xiaoshuijing Miao Peasant Choir in Yunnan. They reflect the unique characteristics of the Miao peasant. This chorus group is composed of more than 40 Miao farmers, who have simple appearances. They have a very pleasant singing voice. It is very touching to the audience. Their singing is based on the Bel Canto's ethnic singing method. Without professional training, the chorus work "Ci Gang" ${ }^{6}$ is complete, and won the judges' high praise in CCTV's competition. It won 13th place. The singing of the Xiaoshuijing chorus group was trained at a young age. This tradition was started by European missionaries in Xiaoshuijing Village and were passed on to the villagers, then from generation to generation. Their voice concept is still very stable. The singing voice is clear. It has a very simple voice. It is a very good representative of the ethnic chorus singing. Therefore, choosing chorus singing is a group's traits, and the best use of this trait is another important way of achieving harmony in chorus singing.

\section{CONCLUSION}

Through the National Youth Singer TV Competition, we have seen the excellent works, performances, singing, and voices of Chinese chorus in recent years. The way to explore harmony in Chinese contemporary choral art is to explore from the perspective of competition. This includes the chorus synergy, chorus singing styles and chorus singing techniques. It shows that Chinese choral art is constantly improving with the development of the times. The artistic appeal of choral art can be enhanced through the different nature of choral art and the possibility of collision. The discussion on harmony in the chorus singing in this article is by no means an exhaustive one. Other discussions on harmony in chorus singing are needed and must continue. However, the author will continue to study the related topics of choral art harmony in future work to make new contributions to the development of research in this field.

\section{REFERENCES}

[1] Yang, F. (2012). Bird's-Eye View of the Development of Chinese Chorus Music in the 20th Century. Music Life, 05, 77-79.

[2] Haken, H. (1997). Synergetics-An Introduction and Advanced Topics. West Germany: Springer-Verlag.

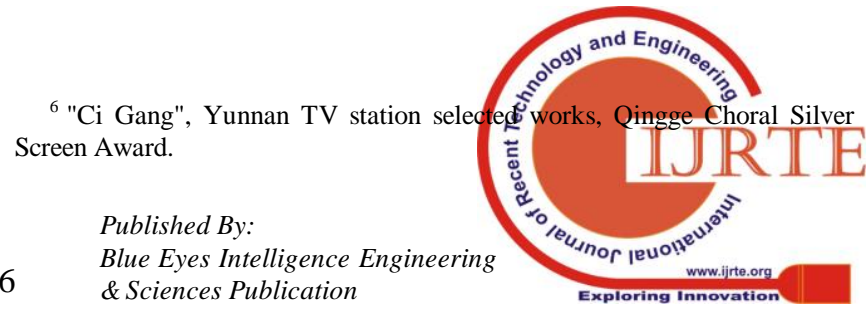


[3] Haken, H. \& Guo. Z. (1983). Higher Collaborative Science. Beijing: Beijing Press.

[4] Xie, L. (2005). The Value Standards of Chinese Contemporary Art. Southern Cultural Forum, 01(13), 70-72. https://doi.org/10.14065/j.cnki.nfwt.2005.01.013

[5] Ma, G. (2002). Chorus. Shanghai: Shanghai Music Publishing House.

[6] Yang, H. (2002). Children's Chorus Training. Beijing: Beijing People's Music Publishing House.

[7] Yang, H. (1982). On the Problem of Chorus Pitch. Journal of the Central Conservatory of Music, 02(006), 20-30.

[8] Shi, Q. (2000). Talking about the pitch training of chorus. Musicology in China, 44-46.

[9] Wang, S. (2006). A Preliminary Study of Singing Language. Journal of Yunnan University of the Arts, 02(014), 67-68.

[10] Yang, H. (2008). Chorus Training. Beijing: Central Conservatory of Music Press.

\section{AUTHORS PROFILE}

My good name is Lu Jingyuan, and my affiliation is with Faculty of Humanities, Arts and Heritage Universiti Malaysia Sabah, 88400kota Kinabalu, Sabah, Malaysia.my area of interest is art and heritage. For any query please contact me at lujingyuan79@foxmail.com

My good name is Lee Chie Tsang Isaiah, and affiliated with Faculty of Humanities, Arts and Heritage Universiti Malaysia Sabah, 88400kota Kinabalu, Sabah, Malaysia. My area of interest is arts and heritage.

${ }^{1}$ Early Chinese choral works: "School Songs" 